\title{
Backup power of a solar power plant through mathematical modelling of processes
}

\author{
V. Onufrei ${ }^{1}$, A. Marchenko ${ }^{1}$ \\ ${ }^{1}$ National Technical University of Ukraine "Igor Sikorsky Kyiv Polytechnic Institute" \\ Corresponding author. E-mail: onufrei@ukr.net
}

Paper received 13.12.18; Accepted for publication 20.12.18.

https://doi.org/10.31174/SEND-NT2018-186VI22-17

\begin{abstract}
In this paper, the justification of the introduction of additional backup power systems is described, as well as the proposed use of the mathematical apparatus of Petri Networks on the example of a solar power station with an adaptive reserve. On the basis of Petri networks, a dynamic model was created in Simulink. The results obtained through the program complex are completely identical with the expectations of the algorithm and the results of the dynamic model. This concept is a basic element of the future construction of a hierarchical reserve model, which will be based on the Petri High-Level Network.
\end{abstract}

Keywords: Petri's network, reserve, quality, unbalance, positions, transitions.

Introduction. The active increase in renewable energy generation (RE) capacity in the Ukrainian power grid puts the reserve energy infrastructure (REI) increasingly stringent requirements for selective switching, as well as the provision of a certain quality of power regulation at the points of dynamic change in electricity generation. The efficiency of the REI is determined by the absence of unacceptable risks associated with the nonlinear functioning of energy objects, the ability to capture power when the failure of certain components of the system and an independent choice reserving the unit. The analysis of solutions to compensate for unscheduled failures of regional capacities in most cases shows that it is the nonproduction of electricity that entails a change in the quality of the energy grid. The distinguishing feature of RE from ordinary generating capacities (for example, NPPs, TPPs) is their definite variability and direct dependence on weather parameters. On the basis of this and the capacity of the reserve, there should be another algorithm that takes into account the costs of compensating the imbalance and chooses the optimal backup path.

American and Chinese energy experts are actively engaged in developing solutions related mathematical modelling. [1]. With the help of dynamic modeling of separate sectors of management of energy complexes, using intelligent digital structures and taking into account the economic feasibility of their application, an optimal prediction of regulation is determined, which gives an idea of the further development of events in the system.

Literature review. The development of forecasting systems and artificial intelligence can be implemented through any mathematical apparatus [11]. At the moment, the most optimal is the Petri net, which can simulate any complex system. Behind the results of works [2,3,13,14], Petri networks can be actively used in high-power engineering, which will allow solving many problems associated with power control, balance and maintaining dynamic stability of the system.

Objective. The purpose of this work is to check the performance of the reserve of a solar power plant, the logic of which was modelled using Petri networks.

Materials and methods. The new generation of power systems [2] has certain intelligent control systems their control systems are typical discrete event systems that are based on a certain prediction of many situations. Thus, its generation, transmission, consumption, reservation and, in general, their control can be modeled and analyzed [3,12] using Petri Networks (PN). The basis of this mathematical apparatus is interconnected by means of transitions $\left(t_{1}, t_{2} . t_{5}\right)$, positions $\left(p_{0}, p_{1} . . p_{6}\right)$, which are the basic components of modelling of energy processes [4]. In addition, using $\mathrm{PN}$ is possible to simulate error predictions and outcomes of certain components of the system. In [5], PN are used to search for logic errors that can be used to identify the very elements of errors, even if protective relays or circuit breakers are faulty in electrical networks based on. For example, [6] presents a method for detecting and localizing irregularities, which occurs during accidents or overloads.

Depending on weather conditions, the generation of RE can vary in a wide range. The most sensitive to changes in natural properties are solar power plants (SPP), the production of which directly depends on the insolation and temperature of the environment. According to [7], in the summer, a surplus is recorded from the projected output of RE, but there are certain local problems with the deficit of these capacities, especially for areas with a "Solar Power Park". Given the fact that the percentage increase in RE in the energy balance of the UES of Ukraine will only increase [8], it is necessary to take into account the significant nonlinearity of power generation in the system with the help of an adaptive reserve, which will allow covering the balance with the least losses for the grid. To simulate such cases, it is advisable to create a conditional model of the SP, which will consist of a solar complex, its reserve, the external power grid, the consumer and a set of control elements (Fig. 1):

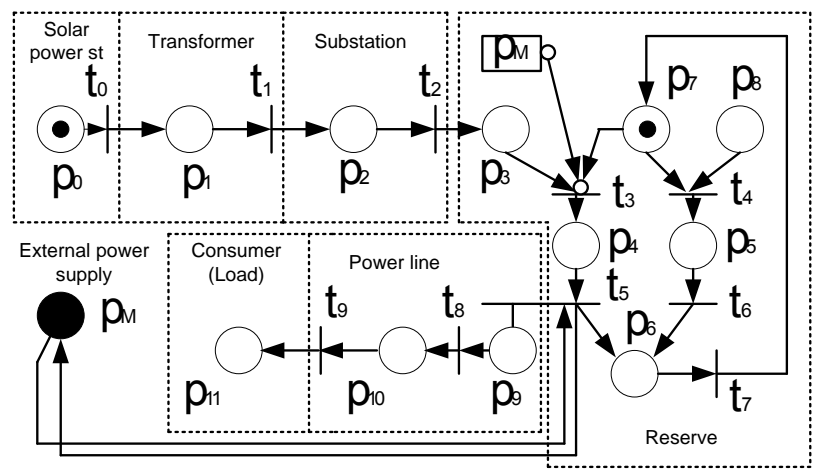

Fig. 1. The model of SPP with reservoir unit in the implementation of PN 
It is worth emphasizing that this option is reserved by an asynchronous loop, which, for semantic reasons, allocates pairs of equivalent components of situations - input and output - or pairs of equivalent initiators and results. The task of constructing a working cycle can be solved, for example, by the "frontal" path - an iterative calculation of the values of the right parts of the system of equations for each of the states of the next tier and comparing them with the values of variables in these states. After detecting the disturbed variables, these variables can be "switched" and according to their new values can be built the status of the next tier, etc. For this kind of execution, a circuit was used to determine the equivalent "result initiator" pair - in fact, it provides selectivity with different types of reductions and thus satisfies the restrictions (1):

$$
\begin{gathered}
S^{m} \subseteq S^{n}\left(I^{* *}\right) \cap S^{n}\left(R^{* *}\right) \\
F^{m}=\left(F\left(I^{* *}\right) \cup F^{\prime}\left(I^{* *}\right)\right) \cap\left(F\left(R^{* *}\right) \cup F^{\prime}\left(R^{* *}\right)\right)
\end{gathered}
$$

when $R^{* *}, I^{* *}$ - set of results and initialization of processes; $F, F^{\prime}$ - markers that specify cause-effect relationships of processes; $S^{m}, S^{n}$ - mode sets.

Depending on the configuration, the model (Fig. 1) can operate in five modes:

- normal operation without compensation, scenario 0 (the tuple $t_{3}$ for entry position $p_{7}$ closed, as the value of the position $p_{3}$ doesn't go beyond the declared output power);

- compensation mode, scenario 1 (power value in position $p_{3}$ went beyond the declared power, capturing this

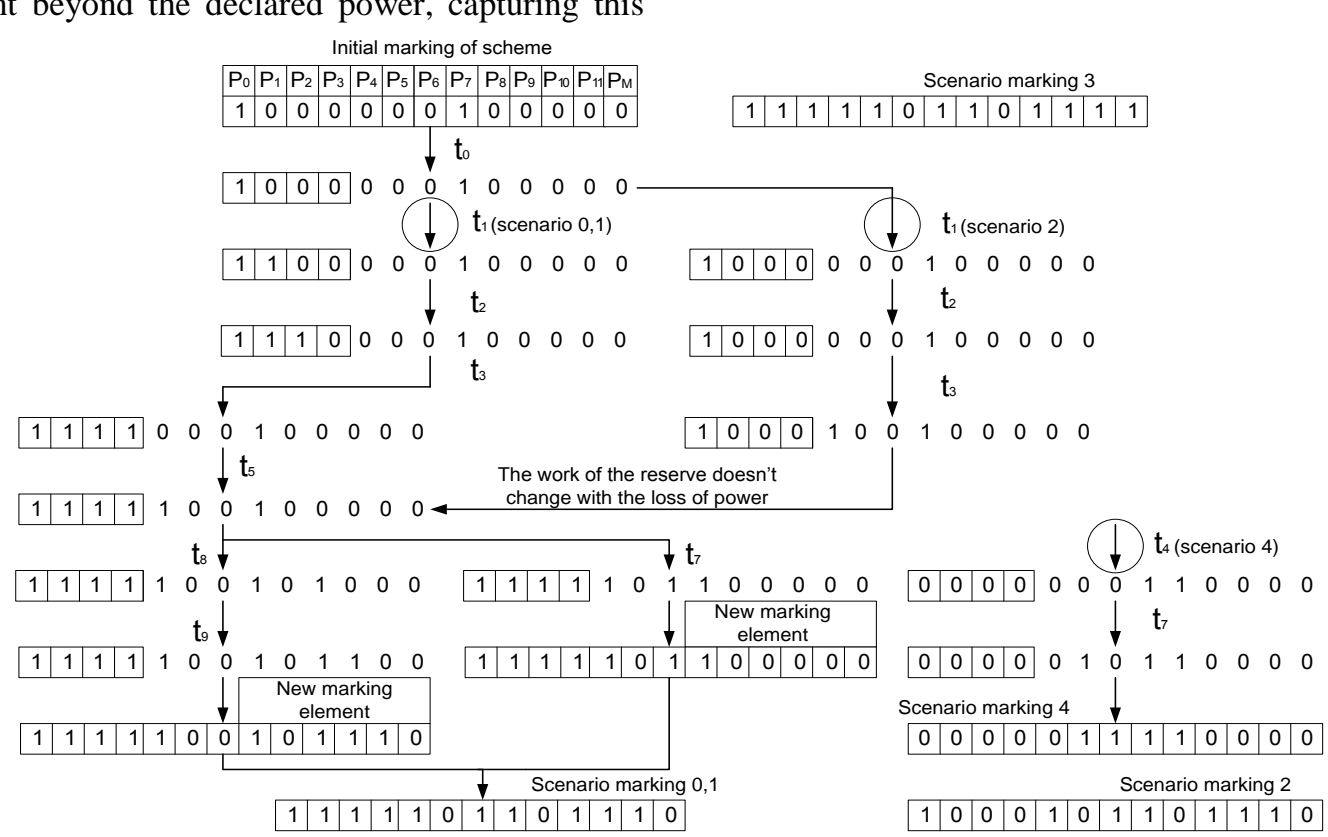

Fig. 2. Diagram of PN-marking for all scenarios of the system of the reserve system of the SPP

Scenarios 0 and 1 have a similar map of work of the system components, but with only one difference - depending on the input value of the marker on the tuple $t_{3}$, the reserve device will correct this discrepancy to the nominal value. Scenario 2 is no different from scenarios 0 and 1 , but the source of power in this variant is no longer SPP, but the reserve itself.

Results and discussion. For a detailed analysis of the processes in the system, transfer the model (Fig. 1) into feature of the tuple $t_{3}$ opens a cell for triggering the entry of the reserve management position $p_{7}$ and thereby tightening power delivery. The system works until it is in position $p_{3}$ the limit value of power won't be restored, thereby returning to the scenario 0 );

- compensation mode, script 2 (in position $p_{3}$ disappears and isn't observed any marker of power, thus giving an understanding of the tuple $t_{3}$ about the disappearance of power from SPP. In this case, the position $p_{7}$ begins to work with the maximum marker of power delivery until the marker occurs in $p_{3}$ and transition to scenario 1 );

- flood mode, scenario 3 (when an external system needs an additional power, a control tuple signal $p_{M}$ enters the tuple $t_{3}$ with a condition for inclusion, followed by a position $p_{7}$ begins to give power in parallel with the issuance of a position $p_{3}$. The tuple $p_{M}$ in normal mode in other modes turned off);

- error mode, scenario 4 (in case of improper operation, reserving the device in position $p_{8}$ there is an arbitrary token and the scheme goes into the state of verification [9]. In this mode, the unit is disconnected from the tuple $t_{3}$ and the marker goes the way $\left\langle p_{8}-p_{5}-p_{6}-p_{7} »\right.$. In this case, when after passing this route $p_{7}=p_{8}-$ the unit is displayed for repair)

A complete description of the processes taking place in the work of certain scenarios is presented in the Petri diagram (Fig. 2), where the first elements $p_{0} . . p_{3}$ are the internal characteristics of the SPP, and $p_{7}, p_{8}-$ reserve condition 


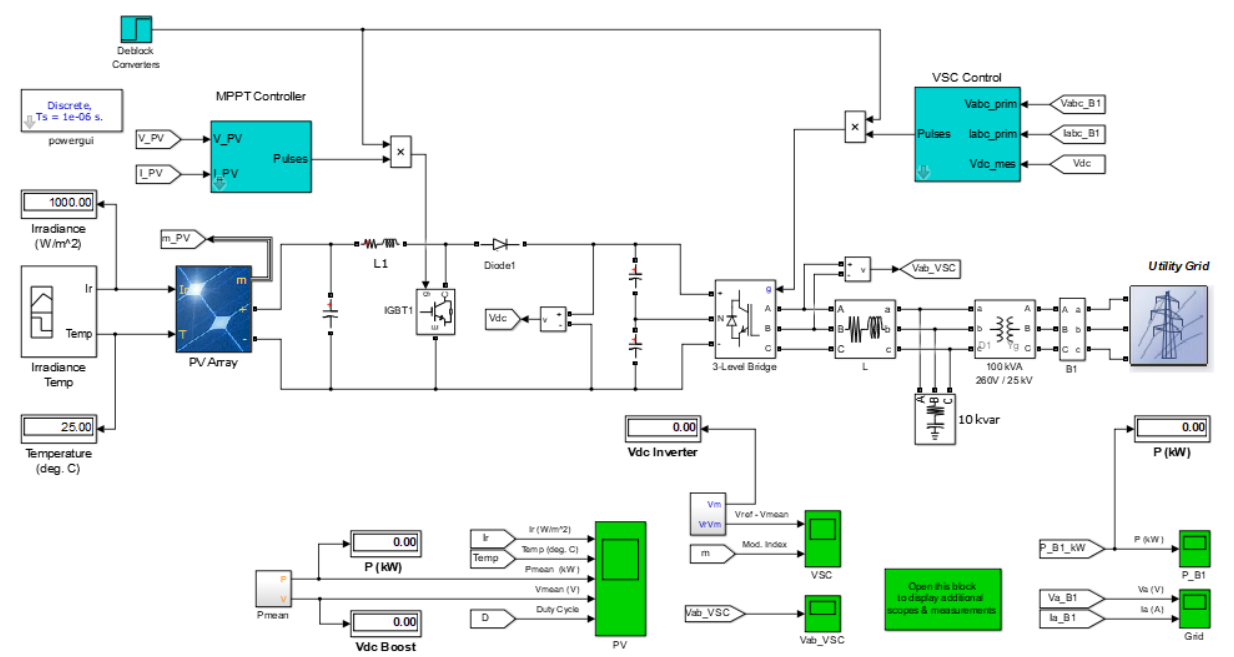

Fig. 3. The model of the generating plot of the scheme $\left(p_{0} . . p_{3}\right)$

The generation system consists of a generating unit of the solar station (PV Array), a voltage controller (VSC Control) and a measuring unit (MPPT Controller), DC circuits and its transformation. The external network is represented by the transmission system (Fig. 4), which includes lines and reserves the body on the side of the medium voltage.

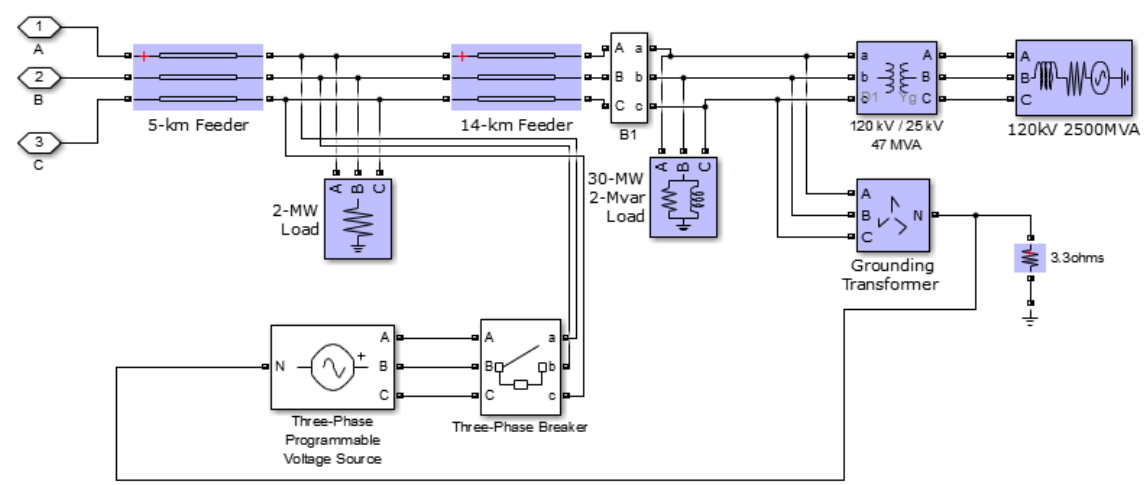

Fig. 4. Model of the transmission part together with the reservoir unit $\left(p_{3} . . p_{6}, p_{M}\right)$

In order to verify the correctness of the logical processes in the system (Fig. 2), scenario 1 was modeled, in which the generation of the solar station varied depending on the parameters of solar radiation in $0,6-1,7$ seconds, as well as with the temperature increase starting from 2,1 seconds. In order to test the operation of the backup device, the circuit with the infinite source of the power system is turned off (Fig. 5):

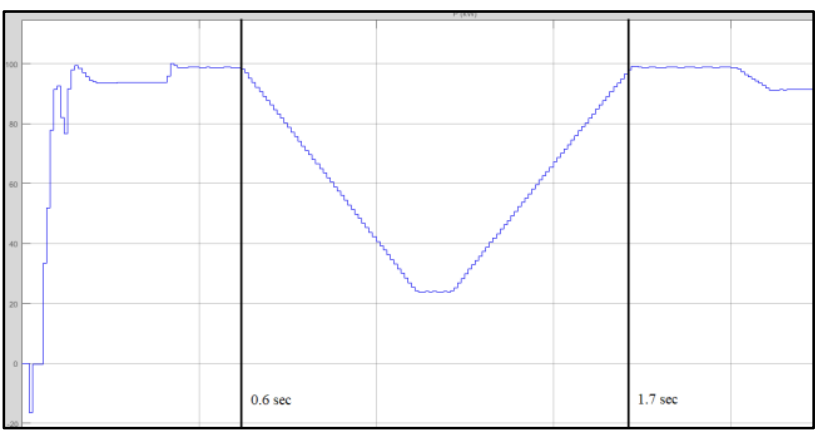

Fig. 5. The generation of SPP with changes in solar activity and temperature

Consider the area of change in solar activity with the use of a reserve unit in the initial and final moments of solar radiation (Fig. 6):

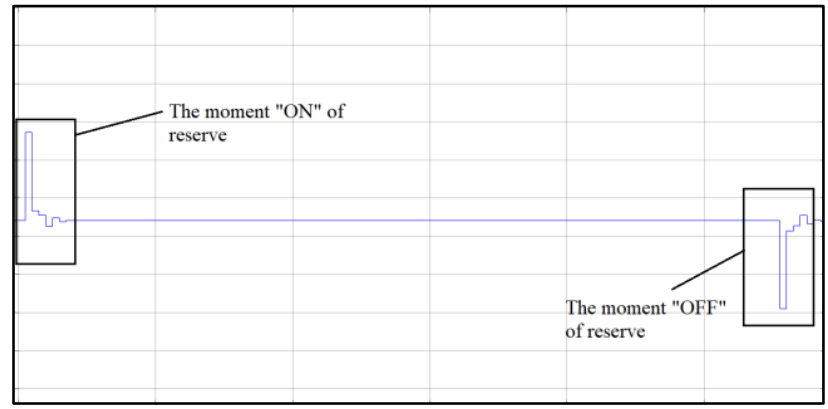

Fig. 6. Power reserve operation at the moment of solar activity change

From Fig. 6 it can be seen that a small jump occurs during on/off times, which is caused by a change in the system mode. During a change in solar activity, a change in power isn't observed, which gives an idea of good modelling and tuning of the backup device.

Conclusions. With a significant increase in the percentage of renewable energy sources, it's necessary to reserve the power data to prevent non-linear unbalances in the system. To accurately interpret the processes occurring in the system, it makes sense to use the mathematical apparatus of Petri Networks, which takes into account not only the asynchronous modes of operation of individual elements but synchronous parametric changes. The simu- 
lation results confirm the effectiveness of the use of dynamic simulation based on the analysis of certain scenari- os for the design of reserve systems and other power systems of the electric power industry.

\section{REFERENCES}

1. Lu, Ning. Power system modeling using Petri nets // A Thesis Submitted to the Graduate, Faculty of Rensselaer Polytechnic Institute in Partial Fullment of the Requirements for the Degree of PhD. New York -2002.

2. H. W. Li, R. X. Lu, L. Zhou, B. Yang, and X. M. Shen, "An efficient Merkle-tree-based authentication scheme for smart grid,” IEEE Syst. J.,vol. 8, no. 2, pp. 655-663, Jun. 2014.

3. G. Ramos, J. L. Sanchez, A. Torres, and M. A. Rios, "Power systems security evaluation using Petri nets," IEEE Trans. Power Del., vol. 25, no. 1, pp. 316-322, Jan. 2010.

4. R. David, H. Alla. Discrete, Continuous, and Hybrid Petri Nets. - Springer, 2010.

5. L. Wang, Q. Chen, Z. J. Gao, and L. Niu, "Knowledge representation and general petri net models for power grid fault diagnosis," IET Gener. Trans. Distrib., vol. 9, no. 9, pp. 866873, 2015.

6. V. Calderaro, C. N. Hadjicostis, A. Piccolo, and P. Siano, "Failure identification in smart grids based on petri net modeling," IEEE Trans. Ind.Electron., vol. 58, no. 10, pp. 4613 4623, Oct. 2011.

7. Characteristics of the workflow from the WED / / DP "Energorinok", - Electronic resource: http://www.gp.er.gov.ua.

8. "The project of the energy strategy of Ukraine until 2035" from 25 February 2017 // the Energy Sector and Industry of Ukraine, - Electronic resource: http://mpe.kmu.gov.ua/minugol/control/uk/doccatalog/list?cu rrDir $=50358$

9. K. Jensen, S. Donatelli, M. Koutny. Transactions on Petri Nets and Other Models of Concurrency. - Springer, 2011.

10. W. Reisig. Elements of Distributed Algorithms: Modeling and Analysis with Petri Nets . - Springer, 2010.

11. Zaitsev D.A. Mathematical models of discrete systems: Tutorial. - Odessa: ONAT them. A.S. Popova, 2004.

12. Joanna Konopko. A Petri Net Model for Distributed Energy System // Warsaw University of Technology, The Faculty of Electronics and Information Technology, Institute of Computer Science. - Warsaw, 2015.

13. Moacyr A. G. de Brito, Leonardo P. Sampaio, Luigi G. Jr., Guilherme A. e Melo, Carlos A. Canesin "Comparative Analysis of MPPT Techniques for PV Applications", 2011 International Conference on Clean Electrical Power (ICCEP), Model: Mathworks (100 kW PV Array).

14. Yarmolyuk OS, Zamkoviy P.O. "Rosrahunok of the vigorous needs of the sleep panel with the untitled information", Staly developed power engineering, 2016. 